\title{
Erratum to: Métastase vertébrale révélatrice d'un adénocarcinome rectal : à propos d'un cas
}

\section{Erratum to: Vertebral metastasis revealing a rectal adenocarcinoma: a case report}

\author{
A. Aissa - A. Bazine $\cdot$ N. Benhmidou $\cdot$ M. Afif $\cdot$ S. El Majjaoui $\cdot$ M. Borahma $\cdot$ B. El Khannoussi $\cdot$ T. Kebdani $\cdot$ \\ N. Benjaafar \\ (C) Lavoisier SAS 2014 \\ Erratum to: $10.1007 / \mathrm{s} 12558-014-0574-8$ \\ Cet article du Journal africain d'hépato-gastroentérologie s'est vu malencontreusement publié dans la liste des articles \\ Online First d'une autre revue, à la suite d'une erreur d'identification numérique. L'éditeur présente ses excuses aux auteurs \\ et aux lecteurs pour ce désagrément.
}

\footnotetext{
A. Aissa $(\bowtie) \cdot$ A. Bazine $\cdot$ N. Benhmidou $\cdot$ M. Afif $\cdot$ S. El Majjaoui $\cdot$

T. Kebdani $\cdot$ N. Benjaafar

Service de radiothérapie-oncologie de l'institut national d'oncologie, Université Mohamed V Souissi, Rabat, Maroc

e-mail : Abdellahaissa@gmail.com

M. Borahma

Service d'hépato gastroentérologie C de l'hôpital Ibn Sina,

Université Mohamed V Souissi, Rabat, Maroc

B. El Khannoussi

Service d'anatomie pathologique de l'institut national d'oncologie,

Université Mohamed V Souissi, Rabat, Maroc
} 